\title{
On Matrix Projective Synchronization and Inverse Matrix Projective Synchronization for Different and Identical Dimensional Discrete-Time Chaotic Systems
}

\author{
Adel Ouannas ${ }^{1}$ and Raghib Abu-Saris ${ }^{2}$ \\ ${ }^{1}$ LAMIS Laboratory, Department of Mathematics and Computer Science, University of Tebessa, 12002 Tebessa, Algeria \\ ${ }^{2}$ Department of Health Informatics, College of Public Health and Health Informatics, \\ King Saud Bin Abdulaziz University for Health Science, Riyadh 11481, Saudi Arabia \\ Correspondence should be addressed to Raghib Abu-Saris; rabusaris@yahoo.com
}

Received 30 September 2015; Revised 19 November 2015; Accepted 7 December 2015

Academic Editor: Qingdu Li

Copyright (C) 2016 A. Ouannas and R. Abu-Saris. This is an open access article distributed under the Creative Commons Attribution License, which permits unrestricted use, distribution, and reproduction in any medium, provided the original work is properly cited.

\begin{abstract}
The problem of matrix projective synchronization (MPS) in discrete-time chaotic systems is investigated, and a new type of discrete chaos synchronization called inverse matrix projective synchronization (IMPS) is introduced. Sufficient conditions are derived for achieving MPS and IMPS between chaotic dynamical systems in discrete-time of different and identical dimensions. Based on new control schemes, Lyapunov stability theory, and stability theory of linear dynamical systems in discrete-time, some synchronization criteria are obtained. Numerical examples and simulations are used to illustrate the use of the proposed schemes.
\end{abstract}

\section{Introduction}

Over the past few decades, chaos synchronization has become an active research subject in nonlinear science and attracted much attention from many fields due its high-potential applications [1-4]. Many powerful methods have been reported to investigate some types of chaos (hyperchaotic) synchronization [5-10] and most of works on synchronization have been concentrated on continuous-time chaotic systems rather than discrete-time chaotic systems. Recently, more attention has been paid to the synchronization of chaos (hyperchaos) in discrete-time dynamical systems [11-19], due to its applications in secure communication and cryptology [20-22].

Up to now, many types of synchronization have been found in interesting chaotic systems in discrete-time such as projective synchronization [23], adaptive-function projective synchronization $[24,25]$, function-cascade synchronization [26], generalized synchronization [27, 28], lag synchronization [29], impulsive synchronization [30], hybrid synchronization [31], Q-S synchronization [32, 33], and full-state hybrid projective synchronization [34, 35]. Among all types of synchronization, projective synchronization (PS) has been extensively considered. In PS, drive and response systems could be synchronized up to a scaling factor $\alpha$. When the scaling constant $\alpha$ is generalized to constant matrix, a new synchronization type appears and is called matrix projective synchronization (MPS). Another interesting problem is the inverse case of MPS, that is, when each response system state synchronizes with a linear combination of drive system states. Obviously, complexity of the scaling factors, in MPS or in IMPS, can have important effect in applications.

In this paper, based on new design control method using Lyapunov stability theory, we would like to present constructive schemes to investigate two new synchronization types: matrix projective synchronization (MPS) and inverse matrix projective synchronization (IMPS) between chaotic dynamical systems in discrete-time. Numerical examples are given to illustrate the effectiveness of the proposed schemes. To be specific, we apply the MPS between discrete-time chaotic systems of different dimensions: the drive 3D Hénonlike map and the controlled 2D Fold map. Furthermore, for the proposed scheme of IMPS, we apply it to 3D generalized Hénon map and the controlled 3D Baier-Klein map.

The rest of this paper is arranged as follows. In Section 2, the problem of MPS between chaotic systems of different dimensions in discrete-time is investigated. In Section 3, 
the IMPS type is proposed for $n$-dimensional chaotic systems in discrete-time. In Section 4, the proposed approaches are applied to two examples and simulations are used to verify the theoretical results derived in this paper. Finally, the paper is concluded in Section 5.

\section{MPS between Different Dimensional Drive System and Response System}

In this section, to study the problem of matrix projective synchronization (MPS), the following drive chaotic system is considered:

$$
X(k+1)=f(X(k))
$$

where $X(k) \in \mathbb{R}^{n}$ is the state vector of the drive system (1) and $f: \mathbb{R}^{n} \rightarrow \mathbb{R}^{n}$.

As a response system, we consider the following chaotic system:

$$
Y(k+1)=B Y(k)+g(Y(k))+U,
$$

where $Y(k) \in \mathbb{R}^{m}, B=\left[b_{i j}\right] \in \mathbb{R}^{m \times m}, g: \mathbb{R}^{m} \rightarrow \mathbb{R}^{m}$, and $U=\left[u_{i}\right] \in \mathbb{R}^{m}$ are the state vector of the response system, the linear part of the response system, the nonlinear part of the response system, and a vector controller, respectively.

Now, we present the definition of matrix projective synchronization (MPS) between the drive system (1) and the response system (2).

Definition 1. The $n$-dimensional drive system (1) and the $m$-dimensional response system (2) are said to be matrix projective synchronized (MPS) if there exists a controller $U=$ $\left[u_{i}\right] \in \mathbb{R}^{m}$ and a given matrix $\Lambda=\left[\Lambda_{i j}\right] \in \mathbb{R}^{m \times n}$ such that the synchronization error

$$
e(k)=Y(k)-\Lambda X(k)
$$

satisfies that

$$
\lim _{k \rightarrow \infty}\|e(k)\|=0
$$

Then, the error system between the drive system (1) and the response system (2) can be derived as

$$
e(k+1)=B Y(k)+g(Y(k))-\Lambda f(X(k))+U .
$$

To achieve MPS between systems (1) and (2), we choose the vector controller $U$ as follows:

$$
\begin{aligned}
U= & -L_{1} Y(k)-g(Y(k))+\Lambda f(X(k)) \\
& +\left(L_{1}-B\right) \Lambda X(k)
\end{aligned}
$$

where $L_{1} \in \mathbb{R}^{m \times m}$ is an unknown control matrix to be determined.

Theorem 2. The drive system (1) and the response system (2) are globally matrix projective synchronized under the controller law (6) if and only if $L_{1}$ is chosen such that the eigenvalues of $\left(B-L_{1}\right)$ lie inside the unit disk.
Proof. By substituting (6) into (5), the error system can be described as

$$
e(k+1)=\left(B-L_{1}\right) e(k) .
$$

Now the result follows immediately for the stability theory of autonomous linear discrete-time systems.

The following result is a corollary of Theorem 2 .

Corollary 3. If $L_{1}$ is chosen such that $\left(B-L_{1}\right)^{T}\left(B-L_{1}\right)-I$ is a negative definite matrix, then the drive system (1) and the response system (2) are globally matrix projective synchronized under the control law (6).

Proof. Although this result is a corollary of Theorem 2, we would like to present an independent proof. Anyhow, once again, by substituting (6) into (5), the error system can be described as

$$
e(k+1)=\left(B-L_{1}\right) e(k) .
$$

To this end, consider a Lyapunov function in the form

$$
V(e(k))=e^{T}(k) e(k) .
$$

Then

$$
\begin{aligned}
\Delta V(e(k))= & e^{T}(k+1) e(k+1)-e^{T}(k) e(k) \\
= & e^{T}(k)\left(B-L_{1}\right)^{T}\left(B-L_{1}\right) e(k) \\
& -e^{T}(k) e(k) \\
= & e^{T}(k)\left[\left(B-L_{1}\right)^{T}\left(B-L_{1}\right)-I\right] e(k) \\
< & 0 .
\end{aligned}
$$

Thus, from the Lyapunov stability theory, it is immediate that the zero solution of the error system (8) is globally asymptotically stable. Therefore, systems (1) and (2) are globally matrix projective synchronized.

\section{IMPS between $n$-Dimensional Drive System and Response System}

In this section, we investigate the problem of IMPS. The drive and the response chaotic systems are in the following forms:

$$
\begin{aligned}
& X(k+1)=A X(k)+f(X(k)), \\
& Y(k+1)=g(Y(k))+U,
\end{aligned}
$$

where $X(k) \in \mathbb{R}^{n}$ and $Y(k) \in \mathbb{R}^{n}$ are the states of the drive system (11) and the response system (12), respectively, $A \in$ $\mathbb{R}^{n \times n}$ is $n \times n$ constant matrix, $f: \mathbb{R}^{n} \rightarrow \mathbb{R}^{n}$ is a nonlinear function, $g: \mathbb{R}^{n} \rightarrow \mathbb{R}^{n}$, and $U \in \mathbb{R}^{n}$ is a controller to be determined.

The definition of inverse matrix projective synchronization (IMPS) for the coupled drive-response chaotic systems given in (11) and (12) is given by the following. 
Definition 4. The drive system (11) and the response system (12) are said to be inverse matrix projective synchronized (IMPS), if there exists a controller $U=\left[u_{i}\right] \in \mathbb{R}^{n}$ and a given matrix $\Lambda=\left[\Lambda_{i j}\right] \in \mathbb{R}^{n \times n}$ such that the synchronization error

$$
e(k)=X(k)-\Lambda Y(k)
$$

satisfies that

$$
\lim _{k \rightarrow+\infty}\|e(k)\|=0 \text {. }
$$

The error system between the drive system (11) and the response system (12) can be derived as

$$
e(k+1)=A X(k)+f(X(k))-\Lambda(g(Y(k))+U) .
$$

To achieve IMPS between systems (11) and (12), we can choose the vector controller $U$ as follows:

$$
\begin{aligned}
U= & -g(Y(k)) \\
& +\Lambda^{-1}\left[f(X(k))+L_{2} X(k)+\left(A-L_{2}\right) \Lambda Y(k)\right],
\end{aligned}
$$

where $\Lambda^{-1}$ is the inverse of matrix $\Lambda$ and $L_{2} \in \mathbb{R}^{n \times n}$ is an unknown control matrix to be determined.

With that in mind, we formulate the following result.

Theorem 5. The drive system (11) and the response system (12) are globally inverse matrix projective synchronized under the control law (16) if and only if $L_{2}$ is chosen such that all eigenvalues of $A-L_{2}$ are strictly inside the unit disk.

Proof. By substituting the control law (16) into (15), the error system can be described as

$$
e(k+1)=\left(A-L_{2}\right) e(k)
$$

Thus, by asymptotic stability of autonomous linear discrete-time systems, it is immediate that all solutions of error system (17) go to zero as $k \rightarrow \infty$ if and only if all eigenvalues of $A-L_{2}$ are strictly inside the unit disk. Therefore, systems (11) and (12) are globally inverse matrix projective synchronized.

\section{Simulation Examples}

In this section, to illustrate the applicability of the theoretical synchronization results derived above, two examples are considered.

4.1. Example 1: MPS between Hénon-Like Map and Fold Map. Here, we consider the Hénon-like map as the drive system and the controlled Fold map as the response system. The Hénon-like map can be described as

$$
\begin{aligned}
& x_{1}(k+1)=1+x_{3}(k)-\alpha x_{2}^{2}(k), \\
& x_{2}(k+1)=1+\beta x_{2}(k)-\alpha x_{1}^{2}(k), \\
& x_{3}(k+1)=\beta x_{1}(k),
\end{aligned}
$$

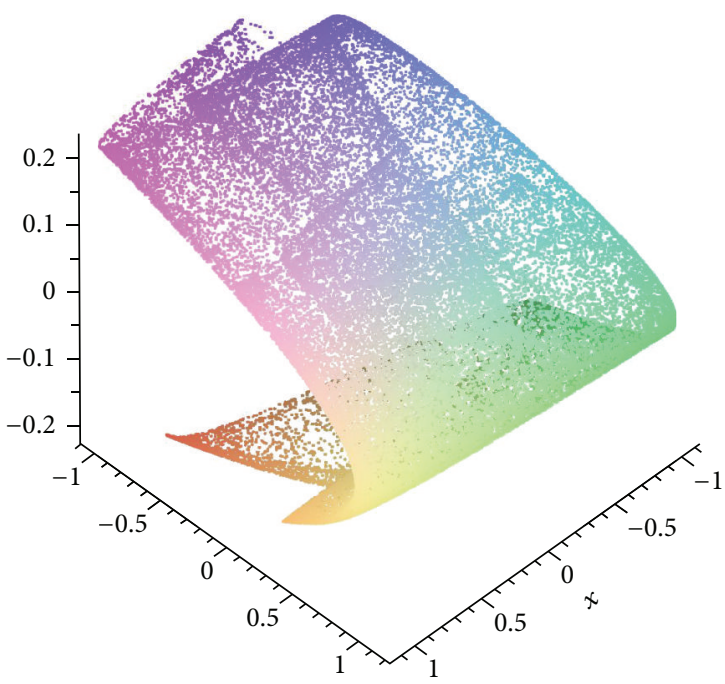

Figure 1: Chaotic attractor of the Hénon-like map when $(\alpha, \beta)=$ $(1.4,0.2)$.

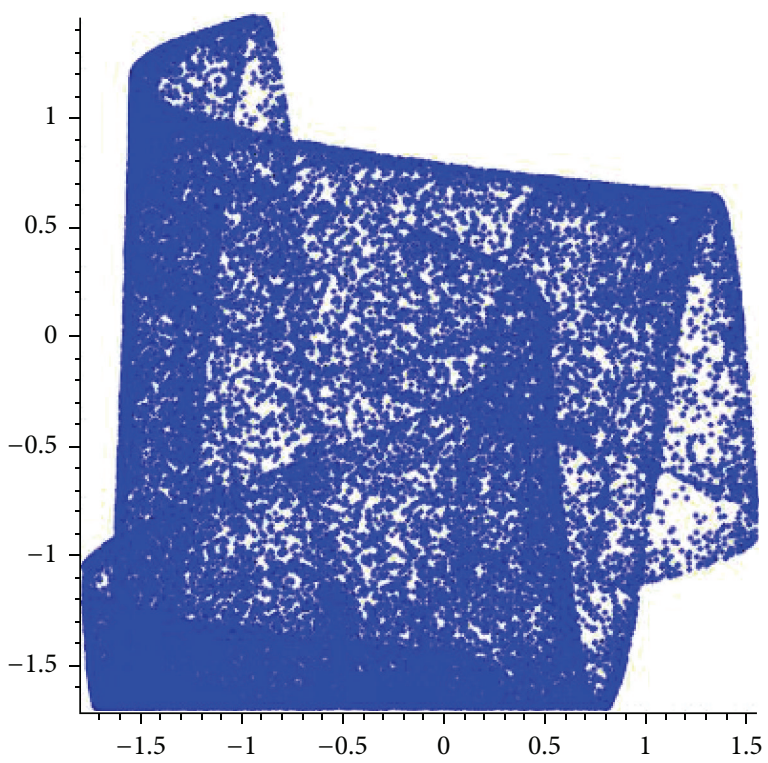

FIgURE 2: Chaotic attractor of the Fold map when $(a, b)=(-0.1$, $-1.7)$.

which has a chaotic attractor, for example, when $(\alpha, \beta)=$ $(1.4,0.2)$ [32]. Using Maple 15.0, the chaotic attractor of the Hénon-like map for $x_{1}(0)=x_{2}(0)=x_{3}(0)=0$ is shown in Figure 1.

The controlled Fold map can be described as

$$
\begin{aligned}
& y_{1}(k+1)=y_{2}(k)+a y_{1}(k)+u_{1}, \\
& y_{2}(k+1)=b+y_{1}^{2}(k)+u_{2},
\end{aligned}
$$

which has a chaotic attractor, for example, when $(a, b)=$ $(-0.1,-1.7)$ [33], where $U=\left(u_{1}, u_{2}\right)^{T}$ is the vector controller. Using Maple 15.0, the chaotic attractor for $y_{1}(0)=y_{2}(0)=0$ of this map is shown in Figure 2. 
In this example, the synchronization criterion presented in Section 3 is applied between systems (18) and (19). Then, quantities $B$ and $g(Y(k))$ are given by, respectively,

$$
\begin{gathered}
B=\left(\begin{array}{ll}
a & 1 \\
0 & 0
\end{array}\right), \\
g(Y(k))=\left(\begin{array}{c}
0 \\
b+y_{1}^{2}(k)
\end{array}\right) ;
\end{gathered}
$$

then the scaling matrix $\Lambda$ and the control matrix $L_{1}$ are selected as, respectively,

$$
\begin{aligned}
\Lambda & =\left(\begin{array}{lll}
1 & 0 & 3 \\
2 & 0 & 2
\end{array}\right), \\
L_{1} & =\left(\begin{array}{cc}
a+\frac{1}{2} & 1 \\
0 & \frac{1}{2}
\end{array}\right) .
\end{aligned}
$$

According to the general control law given in Section 2 by (6), the vector controller can be designed as

$$
\begin{aligned}
u_{1}= & -\left(\frac{1}{2}+a\right) y_{1}(k)-y_{2}(k)+\left(3 \beta+\frac{1}{2}\right) x_{1}(k) \\
& +\frac{5}{2} x_{3}(k)-a x_{2}^{2}(k) \\
u_{2}= & (2-b)-\frac{1}{2} y_{2}(k)+(2 \beta+1) x_{1}(k)+3 x_{3}(k) \\
& -y_{1}^{2}(k)-2 \alpha x_{2}^{2}(k) .
\end{aligned}
$$

Using simple calculations, we can show that $\left(B-L_{1}\right)^{T}(B-$ $\left.L_{1}\right)-I$ is a negative definite matrix. In this case, since the scaling matrix $\Lambda=\left[\Lambda_{i j}\right] \in \mathbb{R}^{2 \times 3}$ has no effect on the eigenvalues of $\left(B-L_{1}\right)$, one can adjust the scaling matrix arbitrarily during control without worrying about the control robustness. Therefore, in this case, systems (18) and (19) are matrix projective synchronized. Using the vector controller (22), the error functions can be written as

$$
\begin{aligned}
& e_{1}(k+1)=-\frac{1}{2} e_{1}(k), \\
& e_{2}(k+1)=-\frac{1}{2} e_{2}(k) .
\end{aligned}
$$

The error function evolution is shown in Figure 3.

4.2. Example 2: IMPS between Generalized Hénon Map and Baier-Klein Map. In this example, we apply the control scheme proposed in Section 3 to achieve IMPS between the drive 3D generalized Hénon map and the response BaierKlein map. The 3D generalized Hénon map can be described as

$$
\begin{aligned}
& x_{1}(k+1)=-\beta x_{2}(k), \\
& x_{2}(k+1)=x_{3}(k)+1-\alpha x_{2}^{2}(k), \\
& x_{3}(k+1)=\beta x_{2}(k)+x_{1}(k),
\end{aligned}
$$

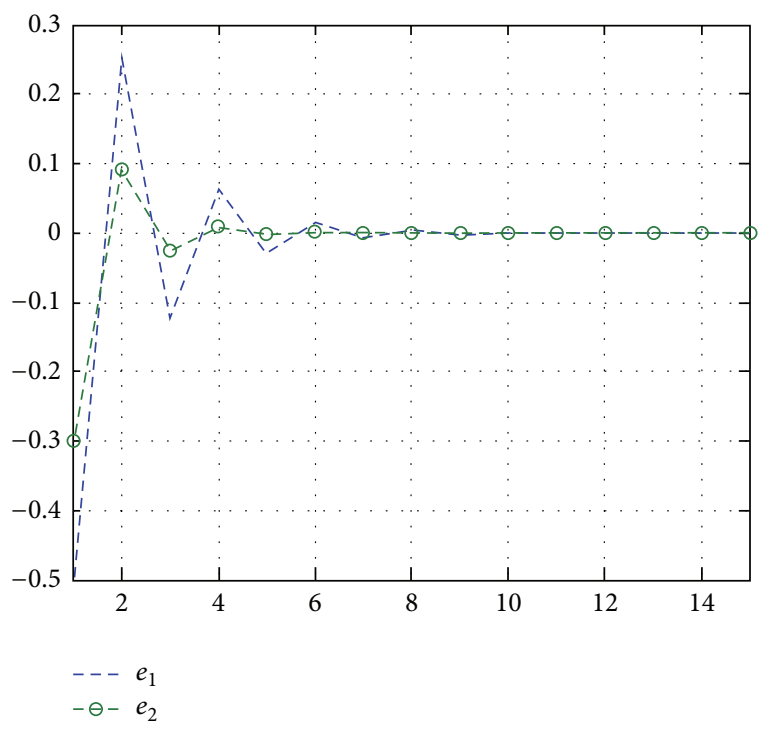

Figure 3: Time evolution of MPS errors between systems (18) and (19).

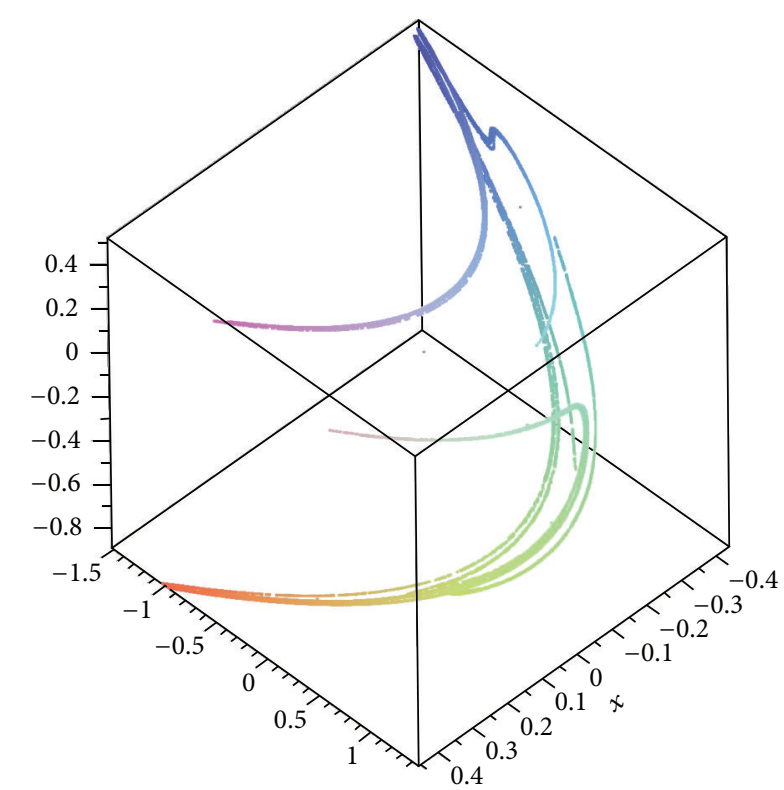

FIGURE 4: Chaotic attractor of the generalized Hénon map when $(a, b)=(1.07,0.3)$.

which has a chaotic attractor, for example, when $(a, b)=$ $(1.07,0.3)$ [32]. Using Maple 15.0, the chaotic attractor of the generalized Hénon map for $x_{1}(0)=x_{2}(0)=x_{3}(0)=0$ is shown in Figure 4.

The controlled Baier-Klein map [36] can be described as

$$
\begin{aligned}
& y_{1}(k+1)=-0.1 y_{3}(k)-y_{2}^{2}(k)+1.76+u_{1}, \\
& y_{2}(k+1)=y_{1}(k)+u_{2}, \\
& y_{3}(k+1)=y_{2}(k)+u_{3},
\end{aligned}
$$




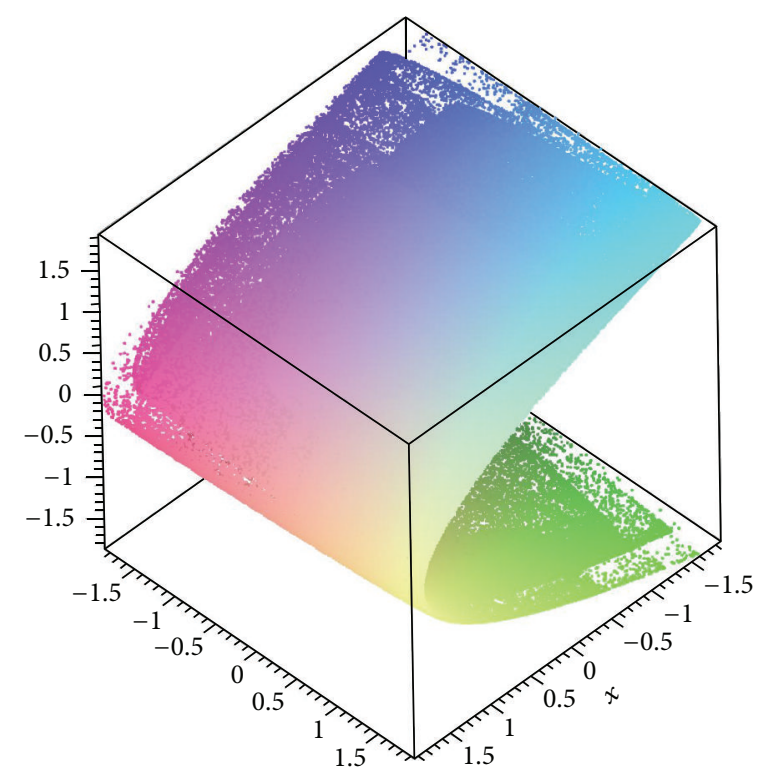

Figure 5: Chaotic attractor of the Baier-Klein map.

where $U=\left(u_{1}, u_{2}, u_{3}\right)^{T}$ is the vector controller. Using Maple 15.0, the chaotic attractor of Baier-Klein map for $y_{1}(0)=$ $y_{2}(0)=y_{3}(0)=0$ is shown in Figure 5 .

According to our approach presented in Section 3, the quantities $A$ and $f(X(k))$ are given by, respectively,

$$
\begin{aligned}
A & =\left(\begin{array}{ccc}
0 & -\beta & 0 \\
0 & 0 & 1 \\
1 & \beta & 0
\end{array}\right), \\
f(X(k)) & =\left(\begin{array}{c}
0 \\
1-\alpha x_{2}^{2}(k) \\
0
\end{array}\right) ;
\end{aligned}
$$

then the scaling matrix $\Lambda$ and the control matrix $L_{2}$ are chosen as, respectively,

$$
\begin{aligned}
\Lambda & =\left(\begin{array}{ccc}
0.5 & 0 & 0 \\
0 & 0.5 & 0 \\
0 & 0 & 0.5
\end{array}\right), \\
L_{2} & =\left(\begin{array}{ccc}
-0.1 & -\beta & 0 \\
0 & 0.2 & 1 \\
1 & \beta & 0.3
\end{array}\right) .
\end{aligned}
$$

In this case, by using the same formula of the control law given by (16), the vector controller can be constructed as follows:

$$
\begin{aligned}
u_{1}= & -1.76+0.1 y_{1}(k)+0.1 y_{3}(k)-0.2 x_{1}(k) \\
& -2 \beta x_{2}(k)+y_{2}^{2}(k), \\
u_{2}=2 & -0.2 y_{2}(k)-y_{1}(k)+0.4 x_{2}(k)-2 \alpha x_{2}^{2}(k),
\end{aligned}
$$

$$
\begin{aligned}
u_{3}= & -0.3 y_{3}(k)-y_{2}(k)+2 x_{1}(k)+2 \beta x_{2}(k) \\
& +0.6 x_{3}(k) .
\end{aligned}
$$

It is easy to show that all eigenvalues of $A-L_{2}$ are strictly inside the unit disk. Therefore, in this case, systems (24) and (25) are inverse matrix projective synchronized. Using controllers (28), the error functions can be described as

$$
\begin{aligned}
& e_{1}(k+1)=0.1 e_{1}(k), \\
& e_{2}(k+1)=-0.2 e_{2}(k), \\
& e_{3}(k+1)=-0.3 e_{3}(k) .
\end{aligned}
$$

The error function evolution is shown in Figure 6.

\section{Conclusion}

In this paper, the problems of matrix projective synchronization (MPS) and inverse matrix projective synchronization (IMPS) in different and identical dimensional discrete-time chaotic systems have been analyzed. Based on nonlinear controllers, Lyapunov stability theory, and stability theory of linear dynamical systems, some synchronization criteria have been obtained and new conditions have been derived for achieving MPS and IMPS. Firstly, to achieve MPS behavior between different dimensional systems, the derived control scheme was proposed by controlling the linear part of the response system. Secondly, to achieve IMPS between identical dimensional systems, the presented control method was proposed by controlling the linear part of the drive system. Numerical examples and simulations were used to verify the effectiveness of the proposed approaches. 


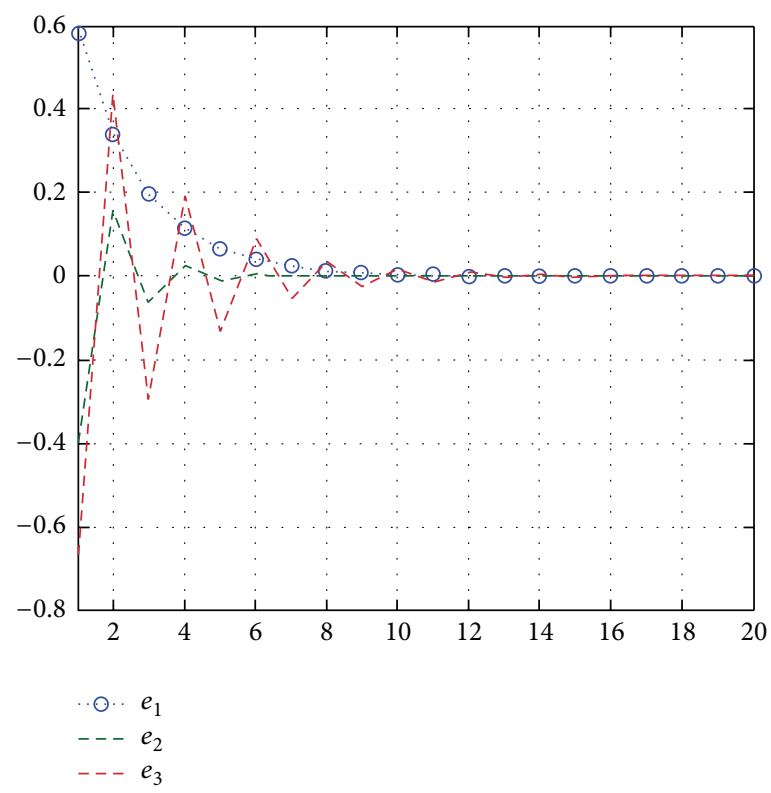

FIGURE 6: Time evolution of IMPS errors between systems (24) and (25).

\section{Conflict of Interests}

The authors declare that there is no conflict of interests regarding the publication of this paper.

\section{References}

[1] R. Roy and K. S. Thornburg Jr., "Experimental synchronization of chaotic lasers," Physical Review Letters, vol. 72, article 2009, 1994.

[2] G. S. Duane, P. J. Webster, and J. B. Weiss, "Co-occurrence of northern and southern hemisphere blocks as partially synchronized chaos," Journal of the Atmospheric Sciences, vol. 56, no. 24, pp. 4183-4205, 1999.

[3] B. Blasius and L. Stone, "Chaos and phase synchronization in ecological systems," International Journal of Bifurcation and Chaos, vol. 10, no. 10, pp. 2361-2380, 2000.

[4] M. Lakshmanan and K. Murali, Chaos in Nonlinear Oscillators: Controlling and Synchronization, World Scientific, Singapore, 1996.

[5] K. S. Ojo, A. N. Njah, and S. T. Ogunjo, "Comparison of backstepping and modified active control in projective synchronization of chaos in an extended Bonhöffer-van der Pol oscillator," Pramana, vol. 80, no. 5, pp. 825-835, 2013.

[6] A. Ouannas, "Chaos synchronization approach based on new criterion of stability," Nonlinear Dynamics and Systems Theory, vol. 14, no. 4, pp. 395-401, 2014.

[7] S. H. Fu and L. J. Pei, "Synchronization of chaotic systems by the generalized Hamiltonian systems approach," Nonlinear Dynamics and Systems Theory, vol. 10, no. 4, pp. 387-396, 2010.

[8] U. E. Vincent and R. Guo, "Adaptive synchronization for oscillators in 6 potentials," Nonlinear Dynamics and Systems Theory, vol. 13, no. 1, pp. 93-106, 2013.

[9] O. I. Olusola, U. E. Vincent, A. N. Njah, and B. A. Idowu, "Global stability and synchronization criteria of linearly coupled gyroscope," Nonlinear Dynamics and Systems Theory, vol. 13, no. 3, pp. 258-269, 2013.
[10] A. Khan and R. Pal, "Adaptive hybrid function projective synchronization of chaotic space-tether system," Nonlinear Dynamics and Systems Theory, vol. 14, no. 1, pp. 44-57, 2014.

[11] H. P. Ju, "A new approach to synchronization of discrete-time chaotic systems," Journal of the Physical Society of Japan, vol. 76, no. 9, Article ID 093002, 2007.

[12] A. Ouannas, "Nonlinear control method of chaos synchronization for arbitrary 2D quadratic dynamical systems in discretetime," International Journal of Mathematical Analysis, vol. 8, no. 53-56, pp. 2611-2617, 2014.

[13] A. Ouannas, "A new chaos synchronization criterion for discrete dynamical systems," Applied Mathematical Sciences, vol. 8, no. 41, pp. 2025-2034, 2014.

[14] A. Ouannas, "Chaos synchronization approach for coupled of arbitrary 3-D quadratic dynamical systems in discrete-time," Far East Journal of Applied Mathematics, vol. 86, no. 3, pp. 225232, 2014

[15] A. Ouannas, "Some synchronization criteria for $N$-dimensional chaotic dynamical systems in discrete-time," Journal of Advanced Research in Applied Mathematics, vol. 6, no. 4, pp. $1-9,2014$.

[16] A. Ouannas, "Synchronization criterion for a class of $N$ dimensional discrete chaotic systems," Journal of Advanced Research in Dynamical and Control Systems, vol. 7, no. 1, pp. 8289, 2015.

[17] A. Ouannas and Z. Odibat, "Generalized synchronization of different dimensional chaotic dynamical systems in discrete time," Nonlinear Dynamics, vol. 81, no. 1-2, pp. 765-771, 2015.

[18] A. Ouannas, "A new synchronization scheme for general 3D quadratic chaotic systems in discrete-time," Nonlinear Dynamics and Systems Theory, vol. 15, no. 2, pp. 163-170, 2015.

[19] A. Ouannas, "A new generalized-type of synchronization for discrete-time chaotic dynamical systems," Journal of Computational and Nonlinear Dynamics, vol. 10, no. 6, Article ID 061019, 2015. 
[20] A. Y. Aguilar-Bustos and C. Cruz-Hernández, "Synchronization of discrete-time hyperchaotic systems: an application in communications," Chaos, Solitons \& Fractals, vol. 41, no. 3, pp. 13011310, 2009.

[21] W. Liu, Z. M. Wang, and W. D. Zhang, "Controlled synchronization of discrete-time chaotic systems under communication constraints," Nonlinear Dynamics, vol. 69, no. 1-2, pp. 223-230, 2012.

[22] R. L. Filali, M. Benrejeb, and P. Borne, "On observer-based secure communication design using discrete-time hyperchaotic systems," Communications in Nonlinear Science and Numerical Simulation, vol. 19, no. 5, pp. 1424-1432, 2014.

[23] Y.-L. Jin, X. Li, and Y. Chen, "Function projective synchronization of discrete-time chaotic and hyperchaotic systems using backstepping method," Communications in Theoretical Physics, vol. 50, no. 1, pp. 111-116, 2008.

[24] Y. Li, Y. Chen, and B. Li, "Adaptive control and function projective synchronization in 2D discrete-time chaotic systems," Communications in Theoretical Physics, vol. 51, no. 2, pp. 270278, 2009.

[25] L. Yin, L. Biao, and C. Yong, "Adaptive function projective synchronization of discrete-time chaotic systems," Chinese Physics Letters, vol. 26, no. 4, Article ID 040504, 2009.

[26] H.-L. An and Y. Chen, "The function cascade synchronization scheme for discrete-time hyperchaotic systems," Communications in Nonlinear Science and Numerical Simulation, vol. 14, no. 4, pp. 1494-1501, 2009.

[27] Z. J. Ma, Z. R. Liu, and G. Zhang, "Generalized synchronization of discrete systems," Applied Mathematics and Mechanics, vol. 28, no. 5, pp. 609-614, 2007.

[28] G. Grassi, "Generalized synchronization between different chaotic maps via dead-beat control," Chinese Physics B, vol. 21, no. 5, Article ID 050505, 2012.

[29] Y. Chai, L. Lü, and H.-Y. Zhao, "Lag synchronization between discrete chaotic systems with diverse structure," Applied Mathematics and Mechanics-English Edition, vol. 31, no. 6, pp. 733738, 2010.

[30] Y. Gao, X. Zhang, G. Lu, and Y. Zheng, "Impulsive synchronization of discrete-time chaotic systems under communication constraints," Communications in Nonlinear Science and Numerical Simulation, vol. 16, no. 3, pp. 1580-1588, 2011.

[31] R. L. Filali, S. Hammami, M. Benrejeb, and P. Borne, "On synchronization, anti-synchronization and hybrid synchronization of 3D discrete generalized Hénon map," Nonlinear Dynamics and Systems Theory, vol. 12, no. 1, pp. 81-95, 2012.

[32] Z. Yan, "Q-S synchronization in 3D Hénon-like map and generalized Hénon map via a scalar controller," Physics Letters A, vol. 342, no. 4, pp. 309-317, 2005.

[33] Z. Y. Yan, "Q-S (complete or anticipated) synchronization backstepping scheme in a class of discrete-time chaotic (hyperchaotic) systems: a symbolic-numeric computation approach," Chaos, vol. 16, no. 1, Article ID 013119, 2006.

[34] G. Grassi, "Arbitrary full-state hybrid projective synchronization for chaotic discrete-time systems via a scalar signal," Chinese Physics B, vol. 21, no. 6, Article ID 060504, 2012.

[35] A. Ouannas, "On full-state hybrid projective synchronization of general discrete chaotic systems," Journal of Nonlinear Dynamics, vol. 2014, Article ID 983293, 6 pages, 2014.

[36] G. Baier and M. Klein, "Maximum hyperchaos in generalized Hénon maps," Physics Letters A, vol. 151, no. 6-7, pp. 281-284, 1990. 


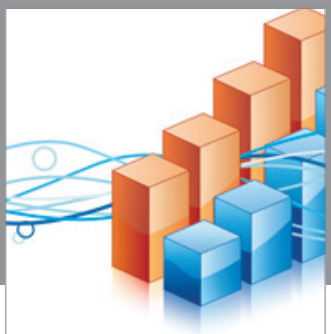

Advances in

Operations Research

vatem alat4

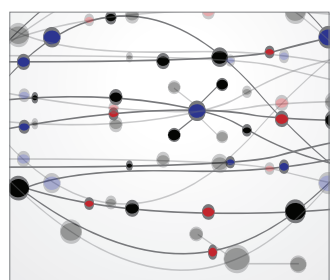

\section{The Scientific} World Journal
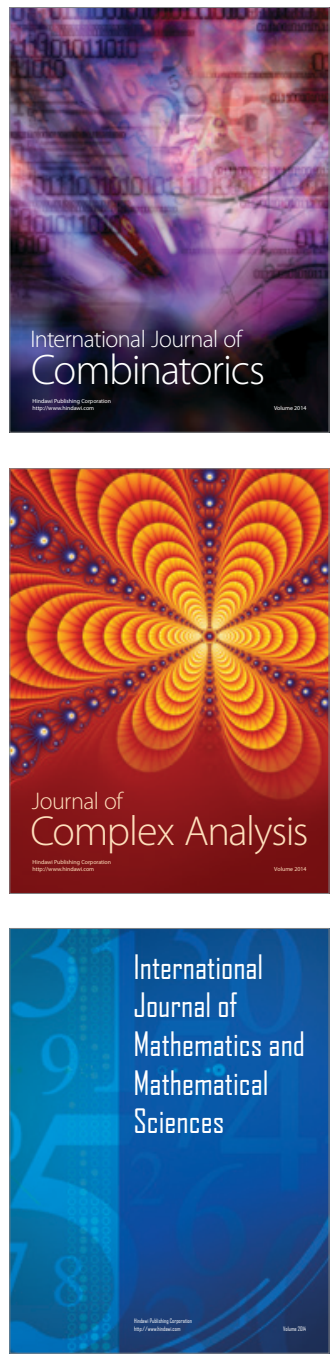
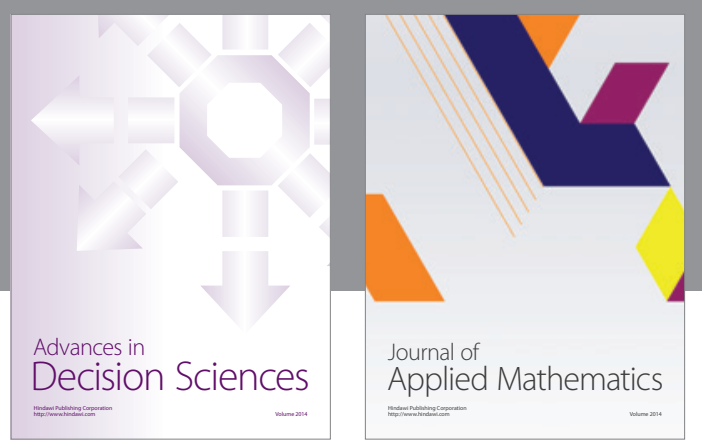

Algebra

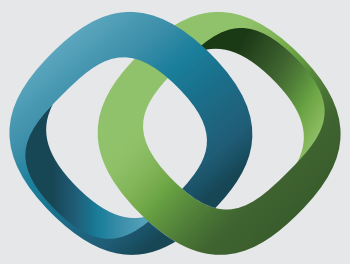

\section{Hindawi}

Submit your manuscripts at

http://www.hindawi.com
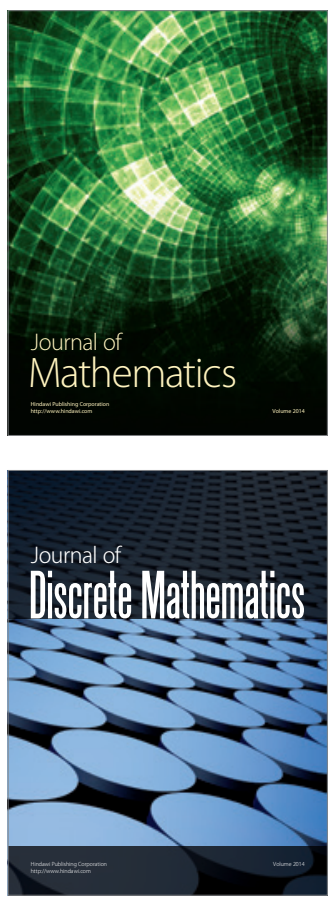

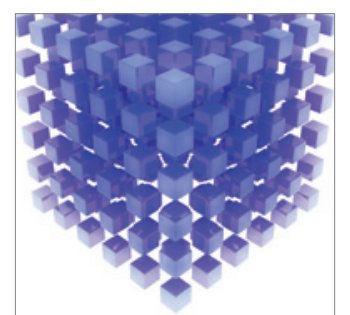

Mathematical Problems in Engineering
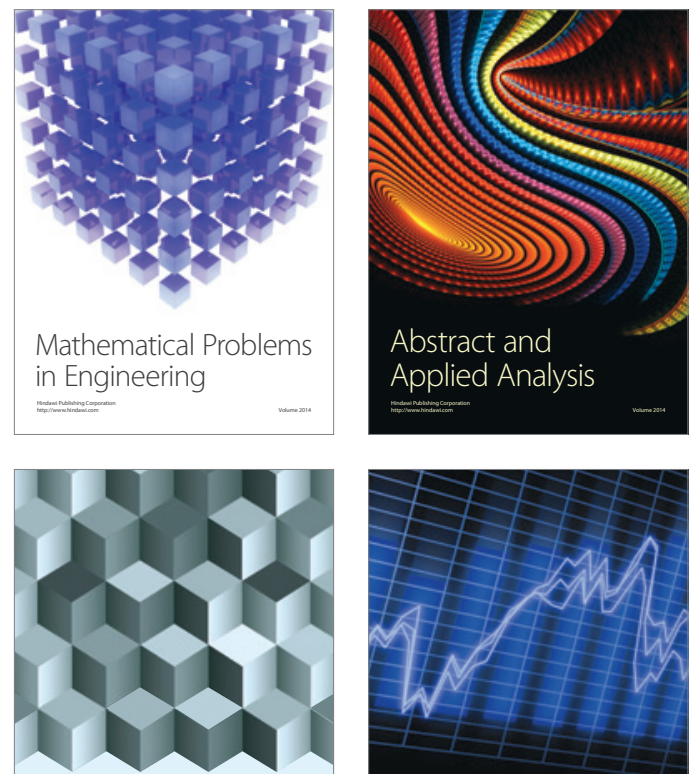

Journal of

Function Spaces

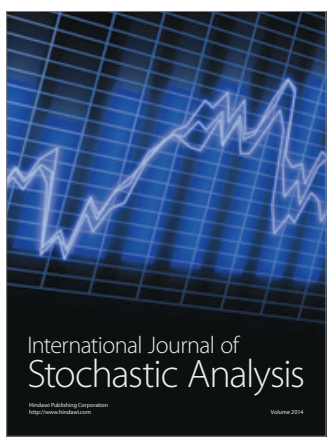

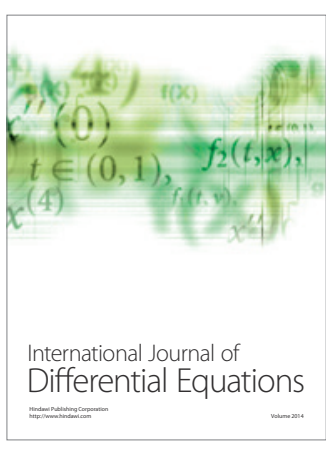
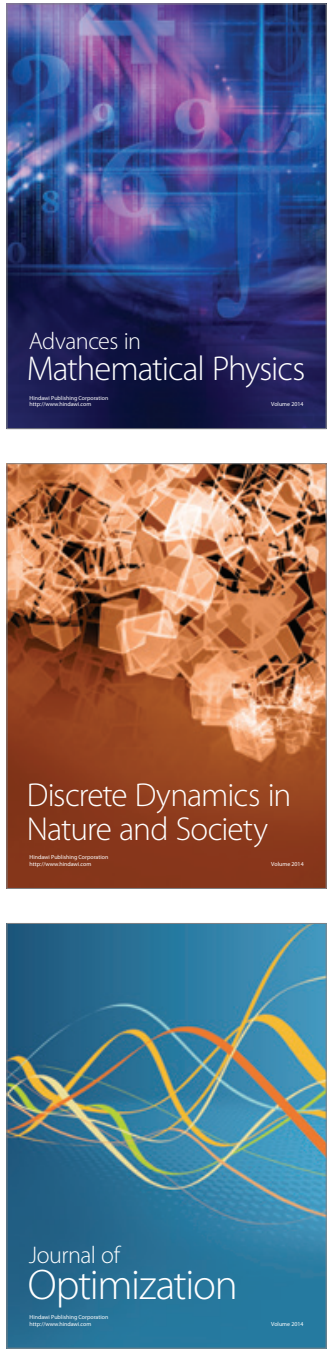